5. Pardi, L. Physiol. Zool. 21, 1-13 (1948).

6. Rohwer, S. Evolution 29, 593-610 (1975).

7. Senar, J. C. in Proc. 22 Int. Ornithol. Congr. (eds Adams, N. J. \& Slotow, R. H.) 1669-1686 (1999).

8. Dale, J., Lank, D. B. \& Reeve, H. K. Am. Nat. 158, 75-86 (2001)
9. Queller, D. C. et al. Nature 405, 784-787 (2000)

10. West-Eberhard, M. J. Proc. Am. Phil. Soc. 123, 222-234 (1979).

11. Paz-y-Miño C, G., Bond, A. B., Kamil, A. C. \& Balda, R. P. Nature 430, 778-781 (2004).

12. Turner, P. E. \& Chao, L. Nature 398, 441-443 (1999).

Condensed-matter physics

\title{
A magnetic pendulum
}

\section{Claude Chappert and Thibaut Devolder}

Where two oppositely magnetized regions meet, there is a so-called domain wall. Under the right conditions, this wall can be made to oscillate like a pendulum, suggesting a new approach to electronics.

$\mathrm{O}$ n page 203 of this issue, Saitoh et al. ${ }^{1}$ provide a beautiful demonstration of a magnetic domain wall oscillating just as a pendulum would do under gravity. A domain wall separates two uniformly magnetized regions and is predicted, at low excitation energies, to behave like a particle with a finite mass. By trapping a single magnetic domain wall in a strip of ferromagnetic material, Saitoh et al. show that they can force the oscillations of the wall inside this potential well, achieving resonant displacements of micrometre length at megahertz frequencies - all with only moderate currents, a key result for possible applications.

The experiment designed by Saitoh et al. is very clever. They patterned a narrow semicircular loop of $\mathrm{Ni}_{81} \mathrm{Fe}_{19}$ wire, $70 \mathrm{~nm}$ wide and $45 \mathrm{~nm}$ deep, onto a silicon substrate. In such a wire of soft ferromagnetic material, dipolar interactions tend to maintain the magnetization parallel to the wire edges. A domain wall will thus separate two areas that are uniformly magnetized antiparallel to each other (Fig. 1a). These 'head-to-head' walls have a nanoscale core that is magnetized perpendicular to the local wire direction. And this is where the semicircular shape comes into play: a magnetic field strong enough to saturate the magnetization of the whole loop, when reduced again to zero, will either leave a single wall in the loop or destroy a pre-existing one, depending solely on the field's orientation (Fig. 1b,c). If a wall is created, increasing the field back to moderate values will tend to trap the wall at the position that keeps its core magnetized parallel to the field direction. The wall will then behave in a manner similar to a simple pendulum, which relaxes towards a stable position under gravity. But here the role of gravity is taken by the magnetic field and hence can be tuned in amplitude and direction gravity itself is not so easy to manipulate!

Applying a periodic force to this'magnetic pendulum' results in an oscillation, whose resonant frequency depends on the effective 'mass' of the domain wall: Saitoh et al. have determined that mass to be $6.6 \times 10^{-23} \mathrm{~kg}$.

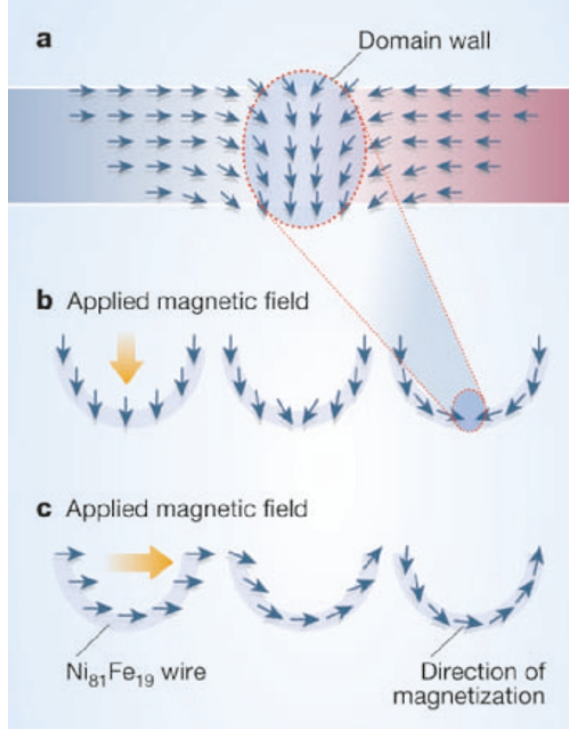

Figure 1 A magnetic pendulum in tunable gravity. a, Where two oppositely magnetized regions meet, there is a domain wall. The arrows indicate the direction of magnetization. Saitoh et al. ${ }^{1}$ have explored the magnetization in a semicircular loop of $\mathrm{Ni}_{81} \mathrm{Fe}_{19}$ wire. $b$, When a magnetic field is applied as shown and then removed, the magnetization of the wire settles into a configuration that includes a single domain wall. c, A magnetic field applied in a perpendicular orientation, however, sets the magnetization such that there is no domain wall. When a domain wall is created, Saitoh et al. have shown that it can be made to behave like a pendulum does under gravity, although in this case the 'gravitational' force - the strength and direction of the magnetic field — can be tuned across a range of values.

The authors have gone further, to investigate the oscillation triggered by passing a sinusoidal current through the wire, using its resonance to explore the interaction between the electrons and the wall. Two types of competing interactions are expected. One is 'momentum transfer', which is basically the mechanical pressure exerted by electrons reflected from the domain wall. The other is 'spin transfer', which arises from the fact that the spins of electrons passing through the domain wall rotate: because total angular momentum is conserved, angular momentum is transferred from the electrons to the magnetization itself, which can trigger movement of the domain wall.

To identify which mechanism is the more relevant, Saitoh et al. take advantage of the different frequency-dependent efficiencies of each transfer process. They find that momentum transfer is a hundred times more efficient than spin transfer in the frequency range of this experiment (from a few to 75 megahertz). This is unexpected in ferromagnetic metals, such as the nickeliron compound used here: most scientists believed that domain walls in such materials are wide enough for flowing spins to rotate adiabatically and track the local magnetization orientation, leading to the dominance of spin transfer. Moreover, by properly choosing the excitation frequency, the threshold current to displace the domain wall could be reduced to a few times $10^{9} \mathrm{~A} \mathrm{~m}^{-2}$, a hundredth of what is observed with direct currents, and well below any heating threshold.

Magnetic domain walls can be considered to be self-assembled stable nanostructures; moreover, they can be created or annihilated by external action. The manipulation of domain walls in stripes has already been proposed as a way of storing information or even performing logic functions ${ }^{2}$. With their result, Saitoh et al. ${ }^{1}$ now provide a low-energy handle on the manipulation of domain walls in complex circuit architectures. And this technique can be fast ${ }^{3}$ and is fairly scalable - the only requirement is to maintain a constant current density as the stripe dimensions are reduced. Saitoh et al. propose some rules of thumb to reduce the required current density even further. In fact, if the ferromagnetic metal were replaced by a dilute magnetic semiconductor, the current density needed to move a wall would already be much smaller ${ }^{4}$.

Conventional (CMOS) electronics is facing major technical hurdles, particularly that of miniaturization. New approaches are needed to reduce energy dissipation and fabrication costs while maintaining the rate of reduction of dimensions, and must be implemented within 15-20 years. This work ${ }^{1}$ could well find application in this direction. Saitoh and colleagues could be a step closer to a new'domain-wall' electronics.

Claude Chappert and Thibaut Devolder are at the Institut d'Electronique Fondamentale,

UMR CNRS 8622, Université Paris-Sud,

91405 Orsay cedex, France.

e-mail: claude.chappert@ief.u-psud.fr

\footnotetext{
1. Saitoh, E., Miyajima, H., Yamaoka, T. \& Tatara, G. Nature 432, 203-206 (2004)

2. Allwood, D. et al. Science 296, 2003-2006 (2002).

3. Nakatani, Y., Thiaville, A. \& Miltat, J. Nature Mater. 2, 521-523 (2003).

4. Yamanouchi, M., Chiba, D., Matsukura, F. \& Ohno, H. Nature
} 428, 539-542 (2004) 